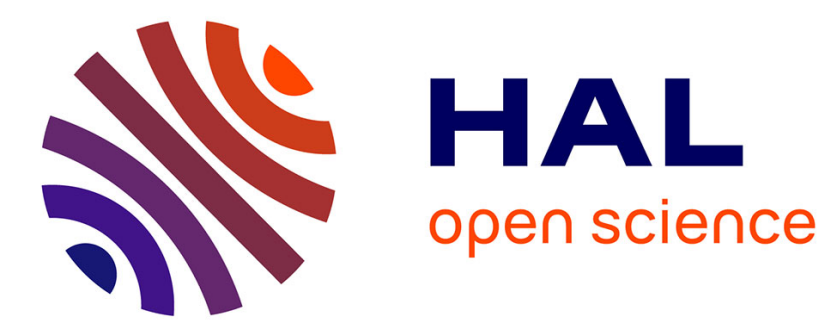

\title{
Drop coalescence and liquid flow in a single Plateau border
}

Alexandre Cohen, Nathalie Fraysse, Christophe Raufaste

\section{To cite this version:}

Alexandre Cohen, Nathalie Fraysse, Christophe Raufaste. Drop coalescence and liquid flow in a single Plateau border. Physical Review E : Statistical, Nonlinear, and Soft Matter Physics, 2015, 91, pp.053008. 10.1103/PhysRevE.91.053008 . hal-01211312

\section{HAL Id: hal-01211312 \\ https://hal.science/hal-01211312}

Submitted on 4 Oct 2015

HAL is a multi-disciplinary open access archive for the deposit and dissemination of scientific research documents, whether they are published or not. The documents may come from teaching and research institutions in France or abroad, or from public or private research centers.
L'archive ouverte pluridisciplinaire HAL, est destinée au dépôt et à la diffusion de documents scientifiques de niveau recherche, publiés ou non, émanant des établissements d'enseignement et de recherche français ou étrangers, des laboratoires publics ou privés. 


\title{
Drop coalescence and liquid flow in a single Plateau border
}

\author{
Alexandre Cohen, ${ }^{*}$ Nathalie Fraysse, and Christophe Raufaste \\ Université Nice Sophia Antipolis, CNRS, LPMC, UMR 7336, Parc Valrose, 06100 Nice, France
}

We report a comprehensive study of the flow of liquid triggered by injecting a droplet into a liquid foam microchannel, also called Plateau border. This drop-injected experiment reveals an intricate dynamics for the liquid redistribution, with two contrasting regimes observed, ruled either by inertia or viscosity. We devoted a previous study [Cohen, PRL 112, 218303 (2014)] to the inertial imbibition regime, unexpected at such small length scales. Here, we report other features of interest of the drop-injected experiment, related to the coalescence of the droplet with the liquid microchannel, to both the inertial and viscous regimes, and to the occurrence of liquid flow through the soap films as well as effects of the interfacial rheology. The transition between the two regimes is investigated and qualitatively accounted for. The relevance of our results to liquid foam drainage is tackled by considering the flow of liquid at the nodes of the network of interconnected microchannels. Extensions of our study to liquid foams are discussed. 


\section{INTRODUCTION}

Liquid foams are dispersions of gas bubbles within a continuous liquid phase. Generally, the liquid is an aqueous solution of surfactants that stabilize the gas-liquid interfaces. Liquid foams are found in numerous applications: food and cosmetics processing, nuclear decontamination, oil recovery [1-3]. Their structure is organized as follows. Liquid films are found at contacts between two bubbles, three bubbles meet along liquid channels called Plateau borders, and four Plateau borders join at vertices. This structure is constantly evolving over time, which might alter the foam properties. One of the aging processes is drainage, namely liquid flows triggered by gravity, other mechanical perturbations, or by capillary suction whenever structural inhomogeneities occur [4]. The drainage of liquid foams under gravity has raised an important interest and has resulted in numerous macroscopic measurements at the foam scale [4-8], as well as a few studies at the bubble scale in the steady regime [9-11]. Drainage was found to occur mostly in the interconnected network of Plateau borders. In all these studies, measurements were satisfactorily accounted for by a model of flow through a porous medium at low Reynolds number. In this context, the interfacial rheology was shown to play an important role on the localization of the flow dissipation [7, 12, 13]. Surfactants leading to tangential stress-free gas-liquid interfaces mainly induce dissipation within the vertices, while surfactants leading to rigid interfaces tend to localize the dissipation within the Plateau borders [7, 13]. Experiments on flows triggered by structural inhomogeneities only are scarce and limited to a few experiments in microgravity $[14,15]$.

We have designed an experiment to study transient flows inside a Plateau border (PB), with capillarity as the only driving force and negligible gravity effects. Our drop-injected experiment consists in releasing a droplet above a single, horizontal PB; the droplet coalesces with the PB as would do a droplet contacting a liquid bath; this triggers a flow of liquid inside the PB, whose dynamics has been systematically studied for various surfactant solutions and experimental conditions.

For surfactants leading to tangential stress-free interfaces, low bulk viscosities and large PBs, we have shown that the perturbation is dispersed through the formation of structures analogous to capillary hydraulic jumps, whose constant velocity was found to be independent of the perturbation size and to scale as the capillary-inertial velocity $c_{0}=\sqrt{\gamma / \rho R_{i}}[16]$, where $\gamma$ and $\rho$ are the surface tension and the density of the surfactant solution, respectively, and $R_{i}$ stands for the radius of curvature of the PB. We have derived a model that points out the analogy with Rayleigh's description of hydraulic jumps[17], and that accounts for the exact shape of the jump. According to the experimental conditions, we may also observe a viscous regime for the liquid redistribution, this time in agreement with the low Reynolds number assumption commonly used in the literature on foam drainage. Here, we present the entire study in order to complete the short report [16] dedicated to the capillary inertial regime.

The manuscript is organized as follows. After the introductory Sec.I, Sec.II describes the experimental setup. Sec. III deals with the coalescence of the droplet with the PB and the subsequent liquid flow, with a detailed description of both the inertial and the viscous regimes. The transition that may occur between the two regimes is also rationalized. Sec. IV is dedicated to three other important issues in the field of liquid foam drainage, namely the imbibition through the vertices, the role of the soap films, and the influence of the interfacial rheology.

\section{MATERIALS AND METHODS}

\section{A. A single-cell model foam}

All our experiments were performed on an elementary foam cell obtained by withdrawing a rigid triangular prism frame (depicted by dashed lines in Fig. 1a) from a surfactant solution. Its two empty triangular bases are equilateral, with edges $3 \mathrm{~cm}$-long, while the length of the prism can be varied from 3 to $12 \mathrm{~cm}$. When dipped into a soapy solution, this frame holds nine soap films; at each end, three films arrange in a tetrahedron, three edges of which being Plateau borders; the three remaining films stretch in between and join in one central, straight Plateau border (PB), ending at a vertex on each side (Fig. 1a, b). The frame is positioned in order for the central PB to be horizontal and for the cross-section of the three films that hold it to take the shape of a Y (Fig. 1c).

Preliminary results (data not shown) showed that the length of the PB, which varies from 1.5 to $11.5 \mathrm{~cm}$ when varying the length of the frame in the above mentioned range, has no effects on the observations reported below; therefore, we kept it constant, equal to $2.5 \mathrm{~cm}$ throughout the present study unless stated otherwise. Because the structure is maintained horizontally, gravity effects on the central PB are reduced and its thickness remains uniform from one vertex to the other. However, the PB thickness decreases slowly with time due to drainage in the vertical direction. A way to compensate for the drainage as well as to vary the PB thickness consists in continuously injecting liquid into the foam cell from one upper-corner of one triangular base of the frame (Fig. 1a). The liquid spreads throughout the structure, which leads to a quasi-uniform and constant thickness for the central PB. The exact flow 
rate through the $\mathrm{PB}$ is not known since most of the liquid leaks downward and does not flow through the central $\mathrm{PB}$. Nevertheless, the PB thickness can be significantly increased by increasing the flow rate of the liquid injected.

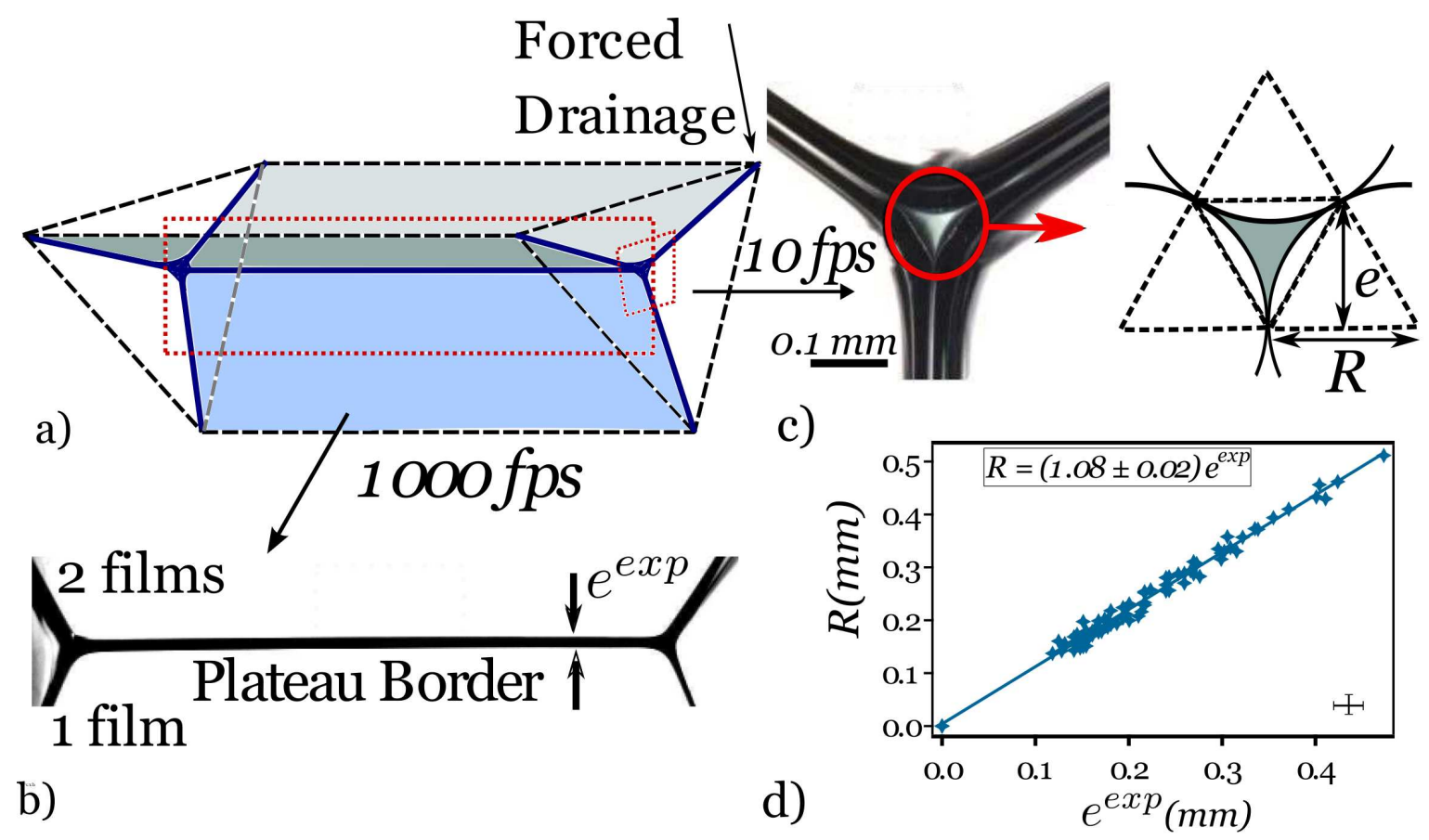

FIG. 1. The single-cell model foam. a) Outline of the experimental setup: the triangular prism frame (dashed black lines) holds the liquid films that join in seven Plateau Borders and two vertices (bold blue lines). The central horizontal PB that spreads between the two vertices constitutes the region of interest of our study (red-dotted quadrangles). b) Side view of the central PB given by the high-speed camera, from which the apparent thickness, $e^{e x p}$, can be measured. c) Longitudinal view. The picture shows the cross-section of the PB seen through one of the vertices (and an adjoining glass plate - see text for details); the sketch depicts the geometry of the PB cross-section and defines the relevant geometrical quantities $R$ and $e$. d) Graph $R$ as function of $e^{e x p}$ given by the calibration step. The two lengths are proportional to an accuracy of $2 \%$ on this example.

Experiments are backlit and filmed from the side by means of a high-speed camera (1000 to $3000 \mathrm{fps}$ ), in order to keep track of the time evolution of the PB (Fig. 1b). The apparent thickness of the PB, $e^{\exp }$, can be measured at any given time; however, this measurement is set-up dependent (the light intensity and the threshold used for the image analysis, inter alia, slightly influence the measured value of the apparent thickness). In order to deduce the real radius of curvature $R$ of the $\mathrm{PB}$ from its apparent thickness, a calibration step needs to be performed before each set of experiments. It consists in coupling simultaneous side and longitudinal views of the PB. The former is given by the high-speed camera, whereas the latter is obtained by use of a standard camera (10 fps) placed on the axis of the PB in order to capture its cross-section geometry through one of the vertices (see [11] for details). To prevent troublesome refractive effects, a glass plate is put in contact with the vertex during calibration; Fig. 1c shows a typical picture thus obtained. The cross-section geometry of the PB is formed by three menisci of same radius of curvature, $R$, in close contact. $R$ is proportional to the height, $e$, of the small triangle drawn on Fig. 1c: $e=\frac{\sqrt{3}}{2} R$. Experimentally, we access $e^{e x p}$ from the side view and $e$ from the longitudinal view simultaneously. We then compute $R$ from $e$ and plot it as a function of $e^{e x p}$ as shown in Fig. 1d. All the calibration curves have been found to be linear to an accuracy of a few percent only. The initial radius of curvature of the Plateau Border, $R_{i}$, varies from $0.1 \mathrm{~mm}$ to $1.3 \mathrm{~mm}$ in the experiments.

\section{B. Droplet addition}

We probe the stability of the PB and study its return to equilibrium by making droplets coalesce with the PB. For this purpose, we release from above small droplets of the same surfactant solution as the single-cell model foam. Due to the Y-shape of the liquid films, the droplet stabilizes on top of the PB after some bounces on the upper liquid films and/or the PB itself. The height at which the droplet is released must not be too large, otherwise the droplet deforms the PB too much; in extreme cases, the droplet can even be penned into a gas bag (forming what is called 


\begin{tabular}{|c|c|c|c|c|c|c|}
\hline solution & glycerol $(\% w)$ & $\rho\left(\mathrm{kg} / \mathrm{m}^{3}\right)$ & $\eta(\mathrm{mPa} . \mathrm{s})$ & $\gamma(\mathrm{mN} / \mathrm{m})$ & $\eta *(\mathrm{mPa} . \mathrm{s} . \mathrm{m})$ & symbol \\
\hline A1 - TTAB 3g/l & 0 & 1030 & 1.0 & 38 & 0.080 & \\
\hline A2 - TTAB 6g/l & 45 & 1100 & 3.4 & 34 & 0.084 & \\
\hline A3 - TTAB 6g/l & 60 & 1140 & 9.2 & 34 & 0.115 & \\
\hline A4 - TTAB 6g/l & 75 & 1150 & 10.4 & 33 & 0.118 & $\checkmark$ \\
\hline A5 - TTAB 6g/l & 80 & 1160 & 12.4 & 33 & 0.161 & \\
\hline A6 - TTAB 6g/l & 85 & 1190 & 27.8 & 32 & 0.172 & $\diamond$ \\
\hline B - SLES+betaine+LAc & 0 & 980 & 0.9 & 20 & 1.115 & $\downarrow$ \\
\hline
\end{tabular}

TABLE I. Properties of the aqueous surfactant solutions at $20^{\circ} \mathrm{C}$.

an anti-bubble), it then passes over the PB and is evacuated along the vertical bottom liquid film without coalescing (images not shown). The radius, $r$, of the droplet is deduced from the photographs afterwards. It varies from $0.2 \mathrm{~mm}$ to $1.8 \mathrm{~mm}$ in the experiments.

\section{Aqueous surfactant solutions}

Two kinds of surfactant solutions were used in the experiments:

- solutions A were obtained by dissolving tetradecyl trimethyl ammonium bromide (TTAB) into deionized water and adding various amounts of glycerol in order to vary the viscosity. The concentration in TTAB was $3 \mathrm{~g} / \mathrm{l}$ for the aqueous solution A1 containing no glycerol, and was raised to $6 \mathrm{~g} / \mathrm{l}$ for the solutions containing glycerol to enhance the stability of the foam cell under study. Solutions A are characterized by tangential stress-free interfaces (high surface mobility limit).

- solution B was one of the surfactant mixtures proposed by Golemanov et al. [18] to vary the dynamics surface properties. This mixture contains sodium lauryl-dioxyethylene sulfate (SLES), cocoamidopropyl betaine (CAPB) and lauric acid (LAc). It was prepared following the protocol reported in [18].

Room temperature was kept to $20^{\circ} \mathrm{C}$ throughout the whole study. The properties of the Solutions $\mathrm{A}$ and $\mathrm{B}$ at $20^{\circ} \mathrm{C}$ are given in Tab. I. The density $\rho$ was measured by weighing a known volume of solution $\left(\mathrm{error}\right.$ of $\left.\pm 50 \mathrm{~kg} / \mathrm{m}^{3}\right)$, the bulk viscosity $\eta$ was determined using a Ubbelohde viscometer (error of $\pm 2 \%$ ) and the surface tension $\gamma$ was measured using the pendant drop method (error of $\pm 1 \mathrm{mN} . \mathrm{m}^{-1}$ ). The dynamics surface properties were characterized through the surface viscosity $\eta^{*}$, which was measured from the growth dynamics of a PB during a T1 process as described in [19] (error of $\pm 5 \%$ ).

In summary, we use Solutions A to study effects of the bulk viscosity, which changes by a factor 30, while the surface mobility does not vary significantly (factor 2 only on the surface viscosity). On the other hand, comparison between Solutions A1 and B makes it possible to investigate the effects of the surface mobility (factor 14 on the surface viscosity) at constant bulk viscosity $(1.0 \pm 0.1 \mathrm{mPa} . \mathrm{s})$.

\section{MAIN STUDY}

\section{A. A Three-stage process}

The perturbation of the Plateau border following the droplet release can be divided into three stages. The first stage is marked by the droplet rebounds and the consequent deformations of the Plateau Border. It ends up with the droplet stabilization on a gaseous lubrication layer. We will not detail this stage here. The second stage deals with the coalescence of the droplet with the PB, which follows the thinning of the lubrication layer under the droplet weight pressure, and its rupture. A third stage deals with the redistribution of the liquid brought by the droplet along the Plateau Border. These last two stages are successively described in the following sub-sections.

\section{B. Coalescence stage}

Once stabilized on the $\mathrm{PB}$, the released droplet rests on a thin layer of air that slowly drains to its point of rupture. This defines the time $t=0$ at which contact is established between the droplet and the PB. From that time, due to 

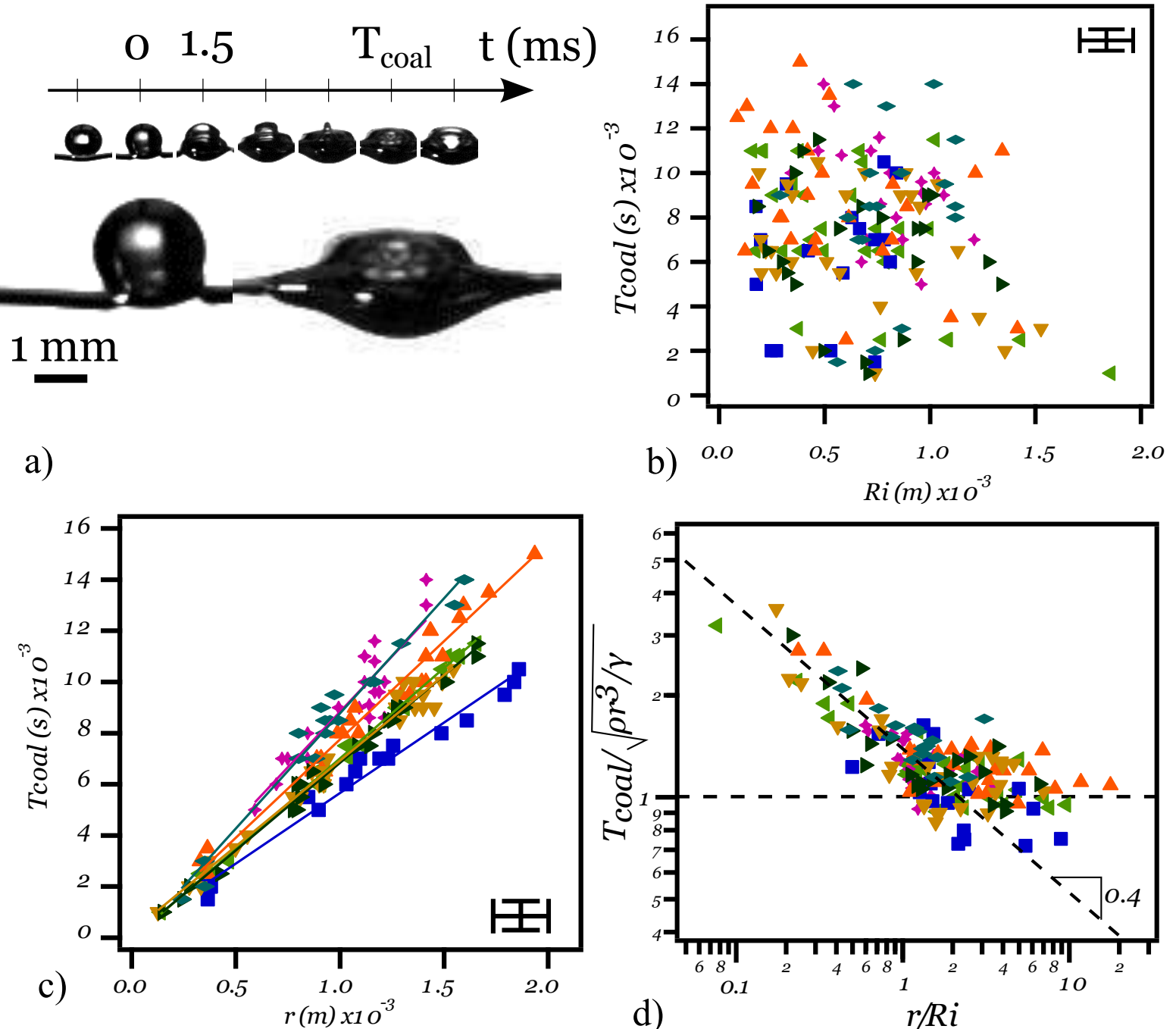

FIG. 2. (a)Image sequence of the coalescence of the droplet with the PB; enlarged pictures of the droplet at $t=0$ and $T_{\text {coal }}$ defined as the coalescence time. (b) Coalescence time as a function of the initial radius of curvature of the PB (see Tab. I for the correspondence between the markers used in Fig. 2b-d and the surfactant solutions). (c) Coalescence time as a function of the droplet radius. Each color straight line is the best linear fit going through zero for the data points represented by markers of the same color. The computed slope equals $[5.5 \pm 0.2 ; 7.7 \pm 0.3 ; 6.7 \pm 0.2 ; 6.9 \pm 0.2 ; 7.1 \pm 0.1 ; 9.0 \pm 0.4 ; 8.7 \pm 0.9]$ for Solution $[A 1 ; A 2 ; A 3 ; A 4 ; A 5 ; A 6 ; B]$, respectively. (d) Ratio of the coalescence time to the capillary-inertial time as a function of the ratio $r / R_{i}$ (logarithmic scales). Dashed black straight lines correspond to $y=1$ and to a slope -0.4 , respectively.

surface tension forces, the area of contact quickly increases; simultaneously, the droplet surface deforms, as can be seen on the image sequence of Fig. 2a. The perturbation of the drop shape spreads upward, the top of the droplet flattens and its curvature eventually changes its sign. In some cases, this process leads to the creation of a daughter droplet, as observed by Thoroddsen and Takehara [20] for droplets coalescing with a liquid bath. The coalescence process is rapid and occurs for the biggest drops within $15 \mathrm{~ms}$ for Solutions A and $20 \mathrm{~ms}$ for Solution B (low surface mobility). Following Thoroddsen and Takehara, we define a coalescence time, $T_{\text {coal }}$, as the time at which the curvature of the top of the droplet comes to zero before changing its sign. Results are reported in Fig. 2. For a given surfactant solution, the coalescence time does not exhibit any clear trend as a function of the radius of curvature, $R_{i}$, of the PB (Fig. 2b), whereas it strongly correlates with the droplet radius, $r$, whatever the $R_{i}$ value (Fig. 2c). As can be expected, the coalescence time increases with the droplet size. This increase is compatible with a linear trend. The slope of the best linear fits equals 8.7 for Solution B and varies from 5.5 to 9.0 for Solutions A; this slight variation (factor 1.6) does not correlate with the variation (factor 30) in bulk viscosity for Solutions A.

The coalescence process is driven by surface tension forces which tend to minimize the total liquid/gas interface area of the system. Experimentally we observe (Fig. 2) that effects of the bulk viscosity and of the initial radius of 
curvature of the PB are small compared to the effects of the droplet radius. Assuming that gravity does not play any role except for bringing together the droplet and the $\mathrm{PB}$, this suggests that the surface tension forces might be balanced by the fluid inertia inside the droplet and that the coalescence time should be compared to the capillary-inertial time $\sqrt{\rho r^{3} / \gamma}$. Without loss of generality, the coalescence time writes

$$
T_{\text {coal }}=\sqrt{\frac{\rho r^{3}}{\gamma}} \cdot \tilde{f}_{\text {coal }}\left(O h, \frac{r}{R_{i}}\right)
$$

where $\tilde{f}_{\text {coal }}$ is a dimensionless function of two dimensionless numbers, the Ohnesorge number, $O h=\eta / \sqrt{\rho \gamma R_{i}}$, which is built by balancing bulk viscous effects with the capillary and inertial ones, and the ratio $r / R_{i}$ of the droplet radius to the $\mathrm{PB}$ radius of curvature.

Fig. 2d shows that $T_{\text {coal }} / \sqrt{\rho r^{3} / \gamma}$ is close to unity, which supports the capillary-inertial mechanism suggested above. A finer analysis shows no correlation of $T_{\text {coal }} / \sqrt{\rho r^{3} / \gamma}$ with $O h$, but exhibits a slight dependency with $r / R_{i}$, which can be described by the following empirical law: $\tilde{f}_{\text {coal }}=(1.3 \pm 0.1)\left(\frac{r}{R_{i}}\right)^{-(0.4 \pm 0.1)}$ if $r / R_{i}<1$ and $\tilde{f}_{\text {coal }} \sim 1$ if $r / R_{i}>1$.

The dependency of $\tilde{f}_{\text {coal }}$ with $r / R_{i}$ is small but reveals a specificity of our $1 \mathrm{D}$ PB-supported geometry when comparing with the coalescence of droplets on a liquid bath (e.g. [20]).

\section{Liquid redistribution stage}

Following the coalescence of the droplet with the $\mathrm{PB}$, the additional liquid reorganizes due to surface tension forces that tend to bring the PB back to a uniform thickness. Fig. 3a, b, and c illustrate the three flow regimes that were identified. Sec. III C is devoted to the first two regimes, the third one observed for Solution B in the low surface mobility limit will be detailed and discussed specifically in Sec. IV C. Fig. 3a depicts the major characteristics of the inertial regime. Two traveling jumps appear on both sides of the coalescing droplet promptly, even before the end of the coalescence process; the velocity of the sharp fronts proves to be constant, and remarkably high, of the order of $1 \mathrm{~m} . \mathrm{s}^{-1}$; upstream of the jumps, the PB is thicker, yet its thickness is uniform, and constant as long as the PB can be supplied in liquid. The viscous regime (Fig. 3b) strongly contrasts with the inertial regime, being much slower, and exhibiting a smooth, bell-like profile for the PB perturbation, which flattens more and more slowly to eventually disappear.

\section{Inertial regime}

The occurrence of a capillary-inertial regime for transient flows in a single foam microchannel was reported in [16, 17] and was unexpected at the small length scales that characterize such a system. This flow regime was described in [16] from measurements performed on two low-viscosity surfactant solutions, one of them being the Solution A1. Here, we investigate it further through the use of Solutions A having higher viscosities. We observed the capillary-inertial regime for bulk viscosities up to $10 \mathrm{mPa} . \mathrm{s}$ (Solutions A1 to A4). The capillary jump geometry that characterizes the capillary-inertial regime is recalled in Fig. 4a. Measurements of the constant, uniform radius of curvature upstream of the jump, $R_{j}$, as well as the jump velocity, $c$, are reported in Fig. $4 \mathrm{~b}, \mathrm{c}$. Note that measurement of the jump velocity requires to take into account the flow that preexists inside the PB due to the continuous supply in liquid; we compute it as the mean value of the velocities $c_{r}$ and $c_{l}$ measured for the jumps propagating in the opposite (on the right-hand side of the $\mathrm{PB}$ perturbation in Fig. 3) and in the same (on the left-hand side of the PB perturbation in Fig. 3) directions of the preexisting flow. Fig. 4b shows that the ratio $R_{j} / R_{i}$ is constant for a given surfactant solution, and does not significantly depend on the viscosity of the solution. The slopes of the best linear fits going through zero for Solutions A1-A4 are reported in Tab. II. The measured jump velocity is found to decrease when the radius of curvature of the $\mathrm{PB}$ increases (Fig. 4c). A power-law interpolation of the data points obtained with Solution A1 leads to $c=(0.0057 \pm 0.0002) R_{i}^{(-0.50 \pm-0.02)}\left(c\right.$ and $R_{i}$ are given in $\mathrm{m} / \mathrm{s}$ and $\left.\mathrm{m}\right)$.

This is consistent with [16], from which $c$ is expected to scale as $c_{0}=\sqrt{\gamma / \rho R_{i}}$. Imposing the exponent of the power-law to be $-1 / 2$ gives the pre-factors reported in Tab. II. The solution viscosity has negligible effects on $c$, as on $R_{j} / R_{i}$. In addition, both quantities prove to be independent on the droplet radius, $r$, as seen on the inserts of Fig. 4b,c. Mass and momentum balance equations when formulated in the specific geometry of the capillary hydraulic jump lead to an intrinsic relation between the imbibition velocity and the jump geometry characterized by the ratio 
a)

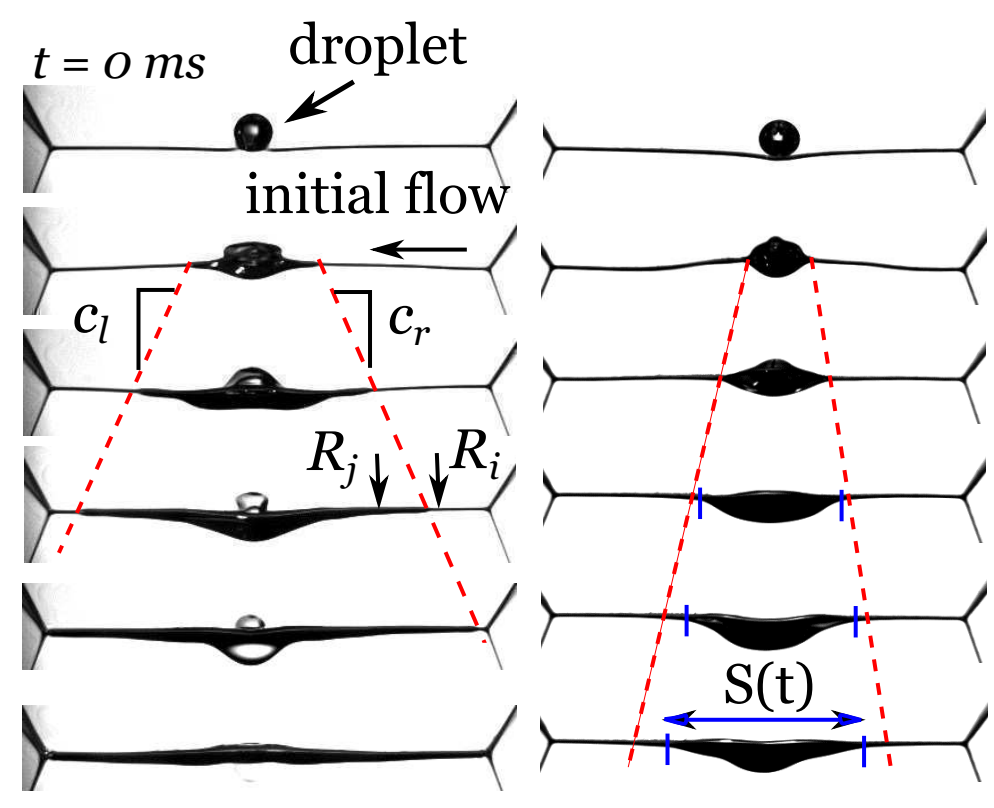

c)
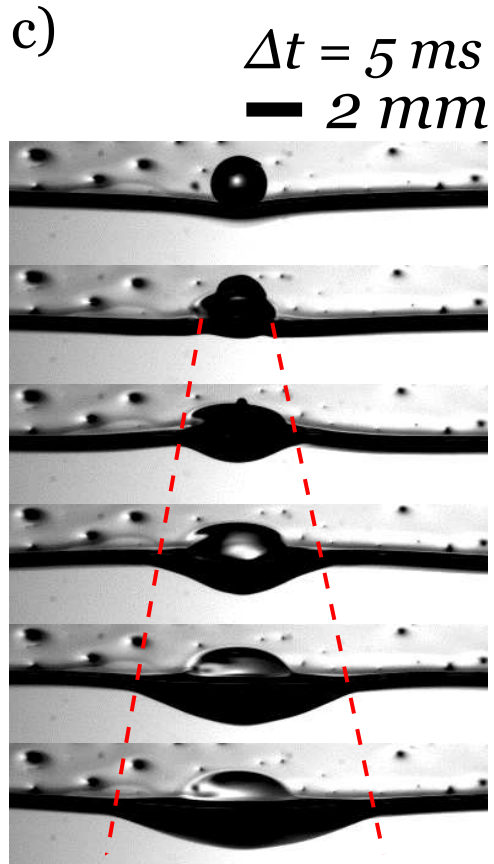

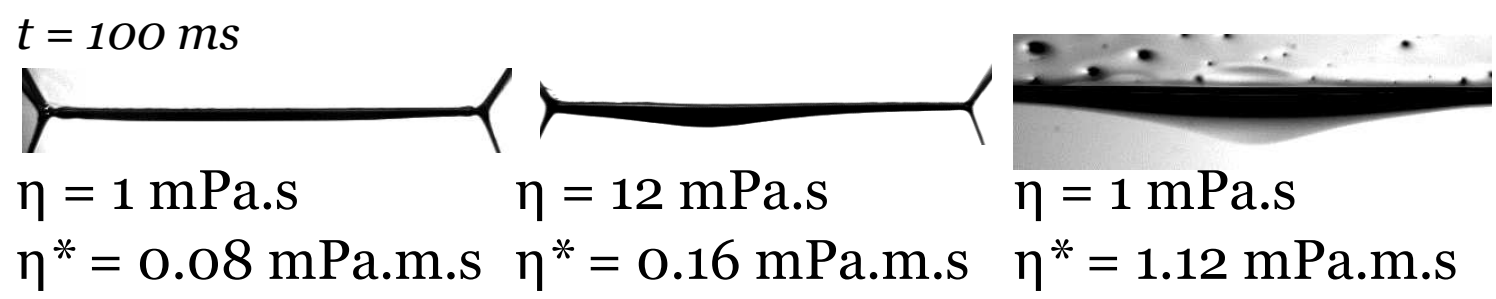

FIG. 3. Three sets of snapshots that illustrate the various regimes observed for the liquid redistribution in a single Plateau border. The first six images of each set were taken every $5 \mathrm{~ms}$ after contact between the droplet and the PB; the seventh image was taken after 100 ms. a) Capillary-inertial regime (Solution A1). A sharp front, which moves at constant, high velocity ( $\sim 0.5$ $\left.\mathrm{m} . \mathrm{s}^{-1}\right)$, separates two regions of constant, uniform thickness of the PB. $c_{r}\left(c_{l}\right)$ stands for the velocity of the front that travels toward the right (left); the preexisting flow goes to the left. The red dashed lines are guides to the eyes to follow the front position. b) Viscous regime (Solution A5). The perturbation slowly spreads along the PB. The evolution can be characterized by measuring $S(t)$ (see Section III.C.2 for details). The red dashed lines are guides to the eyes to compare the actual spreading of the perturbation to a spreading linear in time. c) Liquid redistribution observed in the low surface mobility limit (Solution B). The red dashed lines are guides to the eyes to a constant spreading velocity.

$R_{j} / R_{i}[16]:$

$$
c\left(R_{j} / R_{i}\right)=c_{0} \frac{1}{\sqrt{R_{i} / R_{j}\left(1+R_{i} / R_{j}\right)}},
$$

The measured jump velocity is plotted in Fig. 4d as a function of the theoretical velocity computed using the experimental value of the ratio $R_{j} / R_{i}$ for each surfactant solution (Tab. II). All data points for all four Solutions A1-A4 collapse on the diagonal to a good approximation. In conclusion, this regime does not depend on the bulk viscosity of the surfactant solution, and its dynamics results from a balance between capillary and inertia. These results confirm and extend the conclusions drawn in [16].

\section{Viscous regime}

The dynamics observed during the liquid redistribution stage drastically changes when further increasing the bulk viscosity of the surfactant solution, all other things being equal. Fig. 3b illustrates the relaxation of the PB-with-drop. 




a)



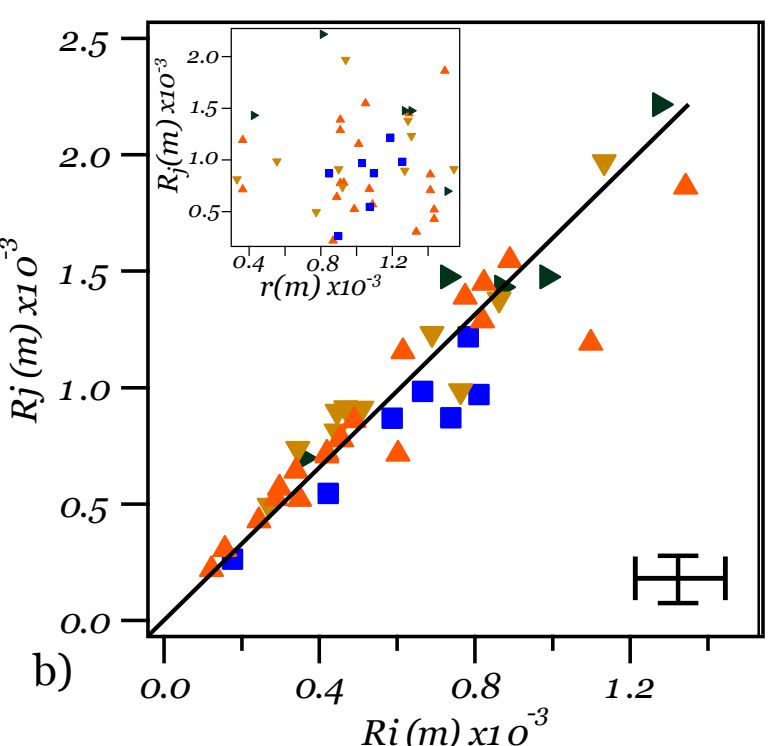



d)

FIG. 4. a) Sketch of the capillary jump geometry. It is characterized by the two radii of curvature, $R_{i}$ and $R_{j}$, of the unperturbed and the perturbed parts of the PB, respectively. The sharp front between these two zones of uniform, constant thickness, moves with a velocity c. b) Radius of curvature upstream of the jump, $R_{j}$, as a function of the radius of curvature downstream, $R_{i}$, for various radii of the coalescing droplets, for Solutions A1- A4 (see Tab.I for markers). The black straight line has a slope equal to 1.7. Insert: $R_{j}$ as a function of the radius $r$ of the released droplet. c) Measured front velocity $c_{\exp }$ as a function of the radius of curvature $R_{i}$ of the PB far from the perturbation [same experiments as in b)] (logarithmic scales). The black straight line on the graph has a slope -0.5. Insert: $c_{\text {exp }}$ as a function of the radius $r$ of the released droplet. d) Measured front velocity $c_{\text {exp }}$ compared to the theoretical velocity derived using the capillary hydraulic jump geometry. The black straight line of slope 1 emphasizes the good agreement between the experimental values and the modeling.

In this regime the central bulge appears to spread smoothly, and the liquid in excess is slowly evacuated into the PB. At any given time, the variation in thickness $e^{\exp }(t)-e^{\exp }(0)$ measured along the longitudinal axis Ox of the $\mathrm{PB}$ yields a bell-like profile as depicted in the insert of Fig. 5a. As stated previously, $t=0$ stands for the beginning of the coalescence stage. We characterize such a dynamics for the liquid redistribution by means of the time-evolution of the spreading distance $S(t)$, which we define as the distance between the two edges of the perturbation (Fig. $3 \mathrm{~b}$ ). In practice, $S(t)$ may be difficult to measure accurately. Strong oscillations of the PB are usually observed after the droplet coalescence, which induces some noise, especially on the basis line of the bell curve. Also, we worked with a $\mathrm{PB}$ as long as possible $(12 \mathrm{~cm})$ to minimize the effects of the vertices which may perturb the flow, however this implies to zoom out and, consequently, to lessen the spatial resolution of the images. We used an algorithm to compute the spreading distance $S(t)$ as the largest distance between two points that belong to the bell curve and whose over-thickness is smaller than $0.2 \mathrm{~mm}$. With the help of this subjective criterion, we were able to obtain the 


\begin{tabular}{|c|c|c|}
\hline Solution & $c / R_{i}^{-1 / 2}\left(\mathrm{~m}^{3 / 2} \cdot \mathrm{s}^{-1}\right)$ & $R_{j} / R_{i}$ \\
\hline A1 & $(57 \pm 2) \cdot 10^{-4}$ & $(1.5 \pm 0.1)$ \\
\hline A2 & $(53 \pm 3) \cdot 10^{-4}$ & $(1.5 \pm 0.1)$ \\
\hline A3 & $(58 \pm 1) \cdot 10^{-4}$ & $(1.7 \pm 0.1)$ \\
\hline A4 & $(59 \pm 2) \cdot 10^{-4}$ & $(1.7 \pm 0.1)$ \\
\hline
\end{tabular}

TABLE II. Results of the curve fitting for Solutions A1-A4 in the capillary-inertial regime.

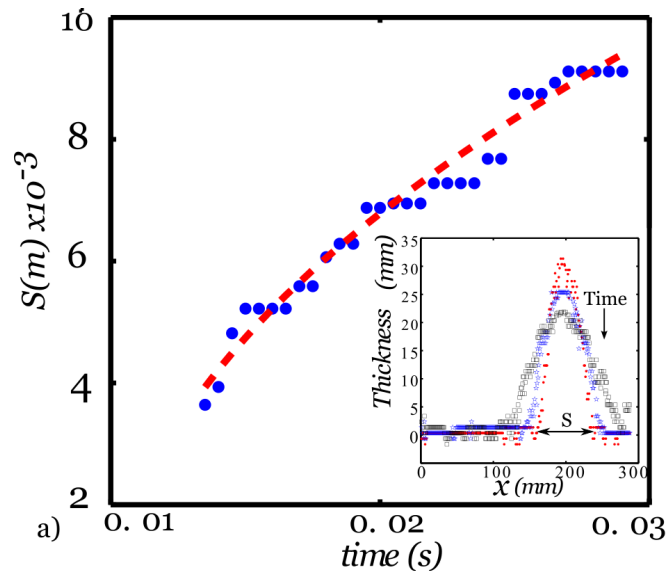

b)
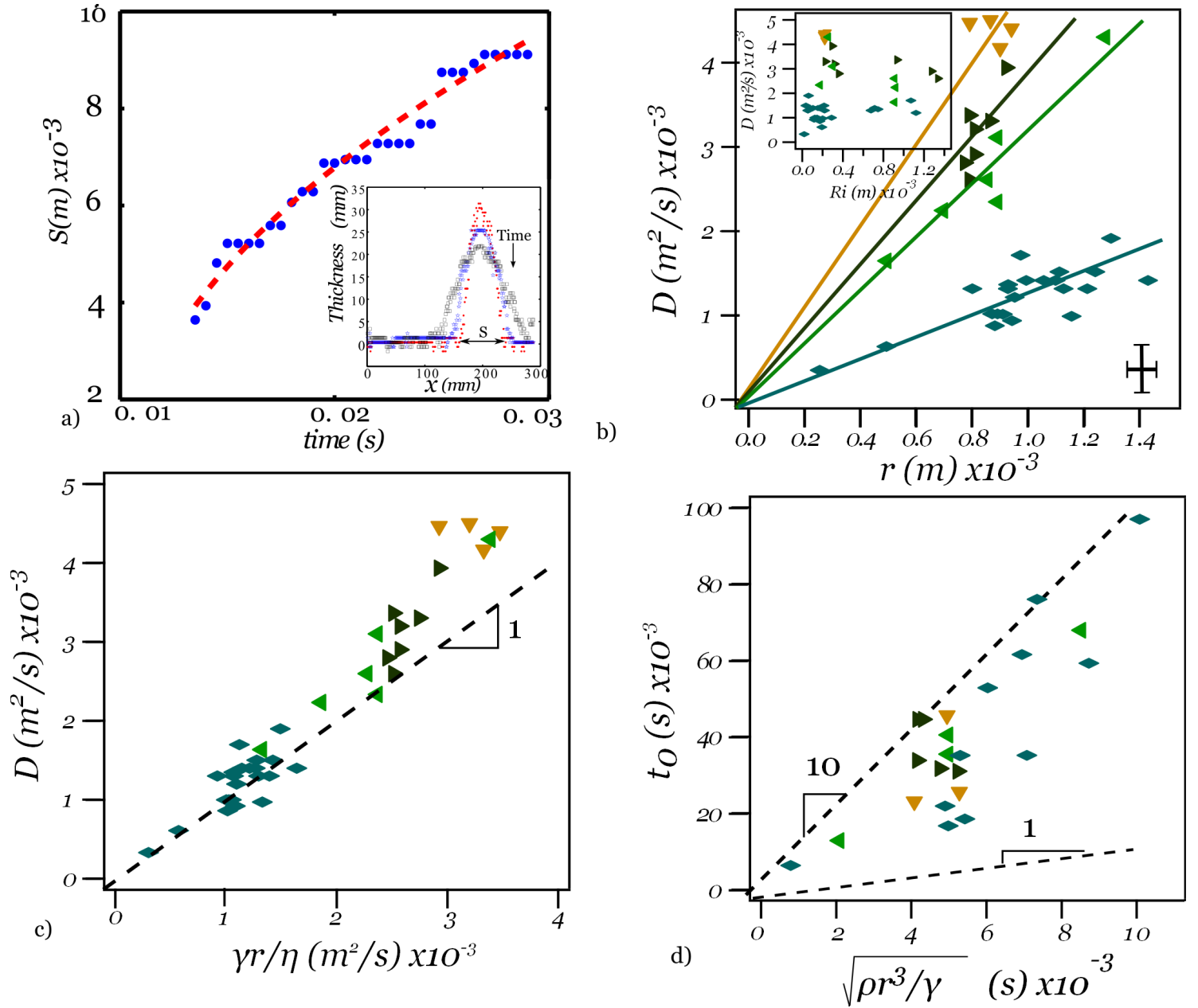

FIG. 5. a) Time evolution of the spreading distance $S(t)$ (blue dots) measured for an experiment performed with the most viscous surfactant solution (Solution A6, $R_{i}=0.7 \mathrm{~mm}, r=1.2 \mathrm{~mm}$ ). The red dashed curve is the $\sqrt{D\left(t-t_{0}\right)}$ interpolation $\left(D=1.3 \mathrm{~m}^{2} \cdot \mathrm{s}^{-1}, t_{0}=12 \mathrm{~ms}\right)$. Insert: bell-like profile of the over thickness of the PB, at three different times. b) Coefficient $D$ as a function of the droplet radius, $r$. Markers refer to Solutions A3 to A6 as stated in Tab. I. The straight color lines are the best linear fits going through zero for the data sets obtained using the various surfactant solutions. Insert: $D$ as a function of the initial radius of curvature of the PB (same experiments). c) $D$ compared to the capillary-viscous diffusion coefficient $\gamma r / \eta$. The black dashed line has a slope 1. d) $t_{0}$ as a function of the capillary-inertial time $\sqrt{\rho r^{3} / \gamma}$. Lines of slope 1 and 10 were drawn for the sake of comparison.

time-evolution of $S$ for each experiment, as plotted in Fig. 5a. Despite a slight residual noise on the experimental data, it appears clearly that the spreading slows down, according to a sub-linear law. We chose to interpolate the $S(t)$ curves by a diffusive law of the form:

$$
S(t)=\sqrt{D\left(t-t_{0}\right)}
$$

where $D$ and $t_{0}$ are two fitting parameters, which have the dimensions of a diffusion coefficient and a time, respectively. We do not claim that the curve fitting leads unambiguously to an exponent $1 / 2$, however a diffusive law is fully 


\begin{tabular}{|c|c|c|}
\hline Solution & $D / \frac{\gamma r}{\eta}$ & $t 0 / \sqrt{\frac{\rho r^{3}}{\gamma}}$ \\
\hline A3 & $(1.1 \pm 0.2)$ & $(6.6 \pm 0.4)$ \\
\hline A4 & $(1.1 \pm 0.1)$ & $(7.2 \pm 0.4)$ \\
\hline A5 & $(1.0 \pm 0.1)$ & $(7.3 \pm 0.4)$ \\
\hline A6 & $(1.0 \pm 0.1)$ & $(6.6 \pm 0.4)$ \\
\hline
\end{tabular}

TABLE III. Numerical values for Solutions A3-A6 in the viscous regime.

compatible with our experimental data and is supported by a simple dimensional analysis as shown below. Scaling laws with exponents $1 / 3$ and $2 / 3$, as found by Piroird and Lorenceau [21] for the imbibition dynamics of oil droplets into a PB, proved to be less consistent with our experimental data than a diffusive law (see Sec. IV.C for a brief discussion on the work by Piroird and Lorenceau, which was performed with rigid surfactant solutions, in relation to ours).

This dynamical regime was observed for Solutions A3 to A6; overall, we obtained D values in the range $0.5 .10^{-3}$ to 4.5.10 ${ }^{-3} \mathrm{~m} . \mathrm{s}^{-2}$, and $t_{0}$ in the range 5 to $100 \mathrm{~ms}$. $D$ is plotted in Fig. $5 \mathrm{~b}$ as a function of the droplet radius, $r$, for all experiments in this regime. $D$ increases with $r$. For any given surfactant solution, the data follow a linear trend; the higher the bulk viscosity of the solution, the smaller the coefficient $D$ and the slope of the linear fit. Insert of Fig. 5b does not show any correlation between $D$ and the initial radius of curvature of the PB.

The experimental results in this regime can be rationalized by considering the dynamics of the liquid redistribution as the result of a capillary-viscous process. The surface tension and the bulk viscosity of the surfactant solution determine the driving force and the damping, respectively. Using the droplet radius $r$, which appears to be the only relevant length scale, we can build a diffusion coefficient, $D=\gamma r / \eta$. Comparison with the experimental $D$-values is shown in Fig. 5c, and the slopes of the best linear fits for Solutions A3-A6 are given in Tab. III. The good agreement between the two quantities supports the capillary-viscous mechanism and a diffusive dynamics. We will thus name this regime the viscous regime. A similar dimensional analysis suggests that $t_{0}$ scales as the capillary-inertial time $\sqrt{\rho r^{3} / \gamma}$ introduced in Sec. III B. Fig. $5 \mathrm{~d}$ plots $t_{0}$ as a function of the capillary-inertial time and Tab. III gives the slopes of the best linear fits for Solutions A3-A6. An average over all the measurements yields a value of $6.9 \pm 0.4$ for the slope.

In conclusion, for large bulk viscosities and for small radii of curvature of the Plateau border, a viscous regime is observed. The liquid inhomogeneity spreads with a smooth thickness profile of the PB. The spreading distance follows a diffusion-like dynamics, whose diffusion coefficient depends on the surface tension, the drop radius and the bulk viscosity. Conversely to the inertial regime, the viscous regime does not depend on the liquid density nor on the PB radius of curvature.

\section{Inertial-viscous transition}

For intermediate bulk viscosities, namely for Solutions A3 and A4, we experimentally observe that the system switches progressively from the inertial regime to the viscous one. At short times, the jump geometry and a constant imbibition velocity are typical of the capillary-inertial regime; at longer times, the thickness profile smoothens and the dynamics slows down, in a way reminiscent of the viscous regime. We illustrate this behavior in Fig. 6a, which displays the time-evolution of the spreading distance for an experiment performed with Solution A3. For each experiment, we define the critical spreading $S_{c}^{\exp }$ as the spreading distance for which the data points depart from the linear start. Note that spreading distances larger than $S_{c}^{e x p}$ are difficult to measure accurately since the rims of the PB perturbation are no longer well-defined fronts. This results in the increasing noise that can be seen in Fig. 6a.

Let's assume that the slower mechanism imposes its dynamics to the system, the transition between the two regimes then occurs when their spreading velocities are similar. The imbibition velocity in the capillary-inertial regime scales as $c_{0}=\sqrt{\gamma / \rho R_{i}}$ (Eq. 2$)$. Its counterpart $c_{\eta}(t)=\frac{1}{2} d S(t) / d t=D / 4 S(t)$ in the viscous regime scales as $\gamma r / \eta S(t)$. Equating the two yields the following scaling for the critical spreading $S_{c}$ :

$$
S_{c} \propto \frac{r}{O h}
$$

where $O h$ is the Ohnesorge number introduced in Section III B. Comparison with experiments is displayed in Fig. 6b. A good agreement is observed, with a prefactor close to $1 / 2$. Note that the range of comparison is restricted due to experimental constraints. We cannot measure values of $S_{c}$ smaller than about one droplet diameter, which is the approximate distance for the imbibition process to become discernible. We were not able to reach large values of $S_{c}$ 

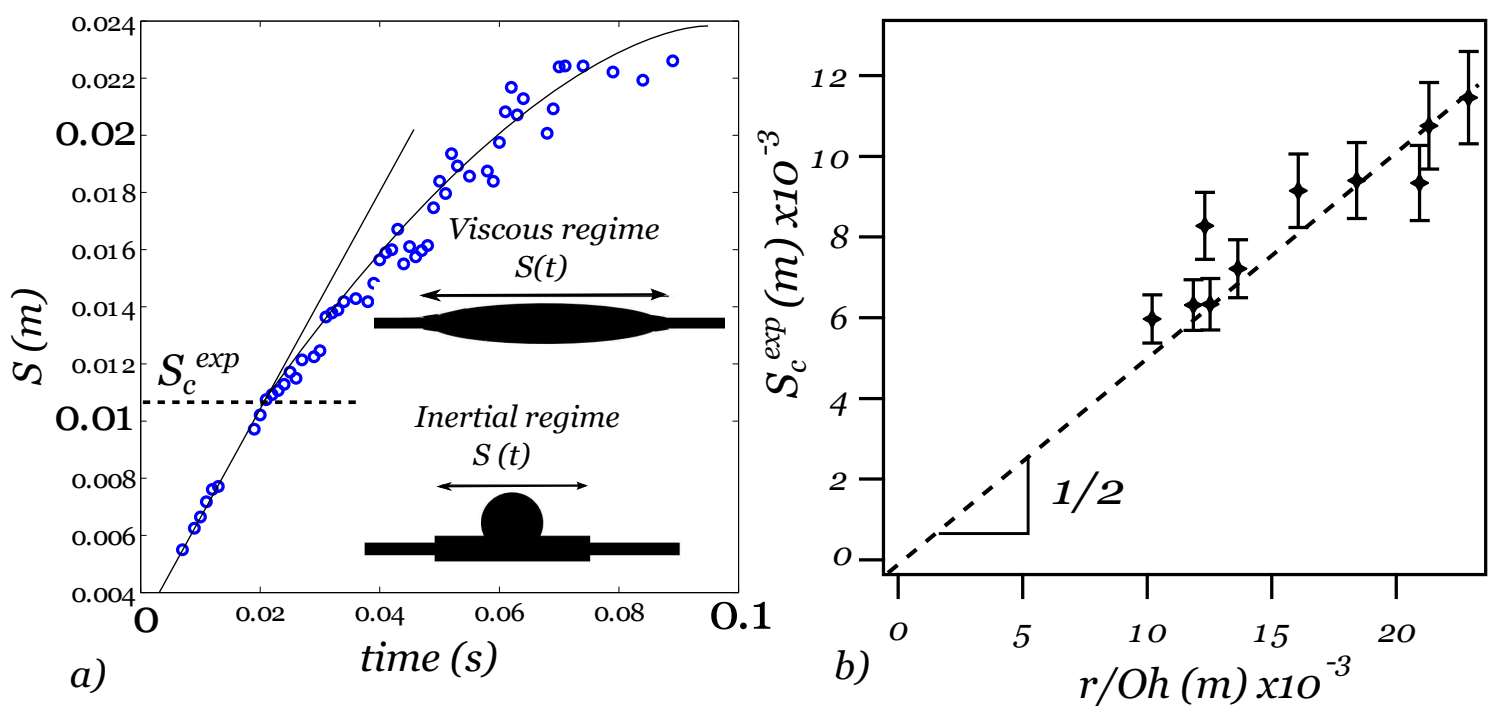

FIG. 6. a) Time evolution of the spreading distance $S(t)$ measured for an experiment performed with Solution A3, $R_{i}=0.7$ $\mathrm{mm}$ and $r=1.2 \mathrm{~mm}$. The thin black lines underline the two consecutive regimes. The critical spreading distance $S_{c}^{\text {exp }}$ at the transition is determined from the experimental curve. b) Critical spreading distance determined experimentally as a function of the ratio $r / O h$. The dashed straight line has a slope $1 / 2$.

neither; this would require a large initial radius of curvature of the $\mathrm{PB}$, and thus a large liquid reservoir to sustain the inertial regime (let's recall that the upstream radius of curvature of the PB is about twice the downstream radius of curvature) over a distance large enough to be measured. We did not succeed in making coalesce droplets with the $\mathrm{PB}$ that were large enough to meet this condition.

The existence of a critical spreading distance related to a transition between the inertial and the viscous regimes, as well as its scaling, refine and amend the criterion given in Cohen et al. [16]. Let's recall that a constant value of $O h$, approximately equal to 0.05 , had been considered to be a limit above which the dynamics was dominated by viscosity and under which the capillary inertial regime was observed, for all values of $r$ and $R_{i}$. However, because our purpose was a careful study of the newly observed inertial regime, we considered its occurrence as certain when the capillary jump had been observed over a distance large enough, typically larger than $5 r$, from the center of the coalescing droplet. This resulted in a biased criterion, which deduces from the one given above by assigning the value of the critical spreading to $10 r$ (twice the arbitrary value that we had chosen for the distance traveled by the jump on one side of the droplet).

A phenomenological criterion to transiently observe the inertial regime in liquid foams can be inferred from what precedes. We may reasonably assume that capillary jumps become discernible from the liquid perturbation that emitted them once the distance they have travelled is larger than about the size of the initial liquid perturbation. Therefore, a minimal condition to detect the inertial regime before the viscous regime takes place, is that the critical spreading is larger than the liquid perturbation size $\left(S_{c} \geqslant 2 r\right)$. This defines a critical radius of curvature of the Plateau borders for the liquid foam: $R_{i}^{c} \propto \frac{4 \eta^{2}}{\gamma \rho}$. For an ideal foam obeying the Kelvin structure, the radius of curvature of the $\mathrm{PBs}$ is related to the bubble radius, $\mathrm{Rb}$, and the liquid fraction, $\phi_{l}: R \propto R_{b} \sqrt{\frac{\phi_{l}}{0.33}}$. These two expressions yields a critical bubble size, of the order of the micrometer for usual aqueous surfactant solutions and dry foams $(\eta=1$ mPa.s, $\rho=1000 \mathrm{~kg} . \mathrm{m}^{-3}, \gamma=30 \mathrm{mN} . \mathrm{m}^{-1}, \phi_{l}=0.01$ ), above which capillary jumps shoud be observed, at least for a short time, when liquid inhomogeneities occur within a liquid foam.

\section{OTHER FEATURES}

\section{A. Flow through a vertex in the inertial regime: influence of the bulk viscosity}

Dissipation in the PB greatly differs according to the flow regime. In particular, shear occurs all over the PB perturbation in the viscous regime; in contrast shear is localized in the narrow zone of the capillary hydraulic jump in the inertial regime, which leads to the faint dissipation evidenced by the independence of the dynamics on the bulk viscosity (see Section III C 1). Let's turn now to what happens at the vertices in the inertial regime. When a 




FIG. 7. a) Snapshots of the vertex before and after being hit by a capillary hydraulic jump (Solution A1). b) Time-evolution of the size of the vertex. Thickest red crosses spot the points that correspond to the snapshots in Fig. 7a. The slope of the thick black straight line defines the rate of increase $V$. The horizontal thin black line emphasizes the saturation at a value $R_{\text {max }}^{v}$.
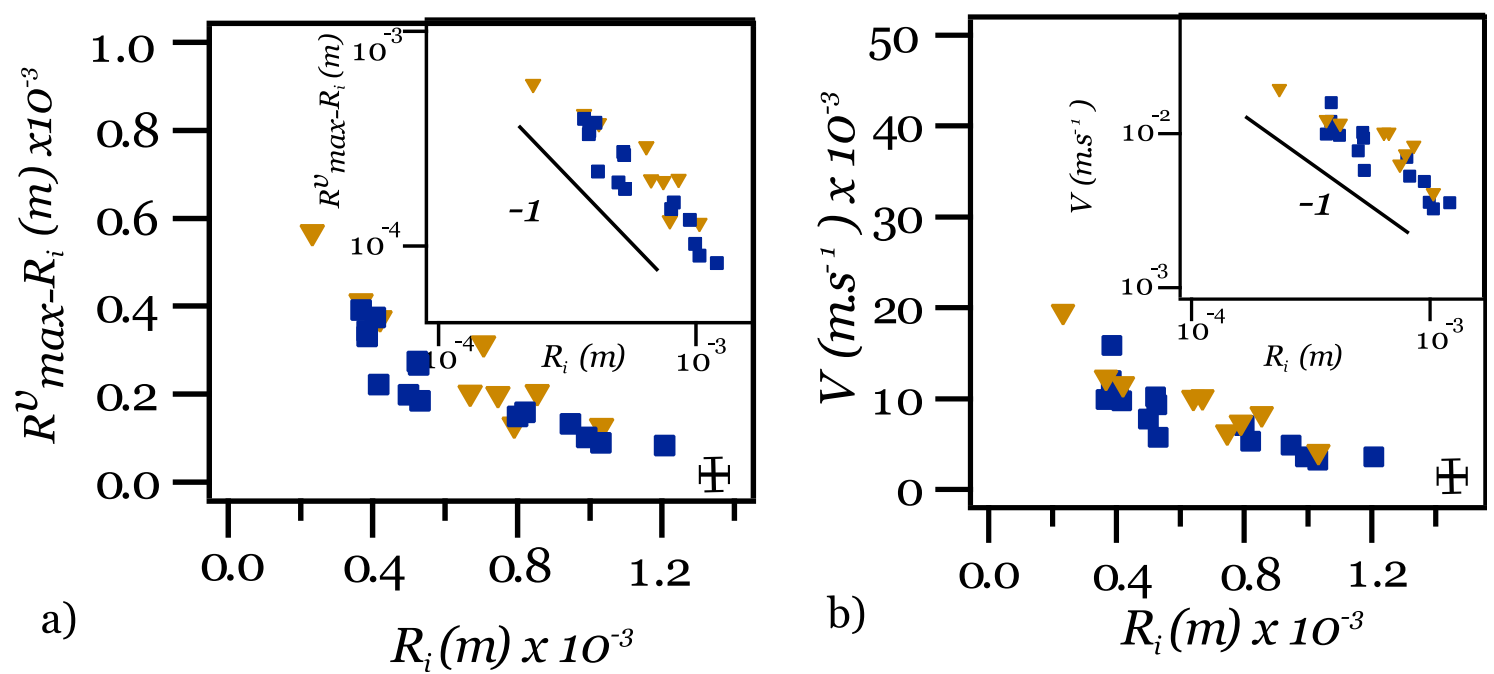

FIG. 8. Characterization of the swelling of the vertex for Solutions A1 (blue squares) and A3 (gold triangles), which differ by a factor 9 in viscosity. a) Maximum increase in size of the vertex, $R_{\max }^{v}-R_{i}$, as a function of its initial size $R_{i}$. Insert: same data in logarithmic scales. The straight thick line has a slope -1. b) Swelling rate of the vertex, $V$, as a function of its initial size $R_{i}$. Insert: same data in logarithmic scales. The straight thick line has a slope -1 .

hydraulic jump reaches a vertex, a much more complicated flow might result from the 3D geometry and dissipation might be affected. To get some insight on this issue, we performed experiments using the same experimental set-up and procedure as before, this time focusing the fast camera on the right-hand side vertex. In order to probe the influence of the bulk viscosity, we used the Solutions A1 and A3, A3 being the most viscous TTAB/glycerol solution leading to the inertial regime; their viscosities are 1.0 and $9.2 \mathrm{mPa} . \mathrm{s}$, respectively; their surface tension and density do not differ significantly. The initial radius of curvature of the PB was varied over one decade. Droplets of large and approximately the same volume were dropped on the PB in order to ensure a large pressure reservoir that supplies the vertex with liquid in an enough durable and steady way to make the analysis possible. Fig. 7a shows snapshots taken after the droplet has coalesced with the PB, when the perturbed part of the PB reaches the vertex and proceeds through it. Once the capillary hydraulic jump has hit it, the vertex swells, and three secondary jumps are created and propagate along the three PBs starting from the vertex; the growth of the vertex eventually saturates; as the liquid reservoir empties, the vertex progressively goes back to its initial size. To quantify the swelling of the vertex observed 
during this process, we measure the radius, $a(t)$, of the circle inscribed in the vertex (see Fig. 7a) as a function of time. For comparison with the PB, we define the size of the vertex $R^{v}$ as the quantity proportional to $a$ that satisfies the relation $R^{v}(t=-\infty)=R_{i}$. Note that, due to Laplace's law, the geometry of the vertex consists of four portions of spheres having the same radius of curvature at equilibrium and a factor 2 exists between the radius of curvature of the PB (or, equivalently, the size of the vertex $R^{v}$ ) and the radius of curvature of the portions of spheres that compose the vertex interface. A typical measurement is displayed in Fig. 7b: $R^{v}$ increases from its initial value $R_{i}$ to a constant value $R_{\max }^{v}$, at a roughly constant rate $V$, of the order of $10^{-2} \mathrm{~m} \cdot \mathrm{s}^{-1}$. Deflation is much slower. A linear adjustment gives rates about $10^{-3} \mathrm{~m} \cdot \mathrm{s}^{-1}$ (data not shown).

The increase in size of the vertex, $R_{\max }^{v}-R_{i}$, and the swelling rate $V$ are plotted as a function of the initial radius of curvature $R_{i}$ in Fig. 8a and b respectively, for two sets of experiments performed with Solutions A1 and A3. Both quantities decrease when $R_{i}$ increases, and the data are compatible with $1 / R_{i}$ laws. Remarkably, the two sets of experimental data superimpose on these plots: the solution viscosity appears not to be relevant. Imposing the exponents of the power laws to be -1 , the best fits give $R_{\max }^{v}-R_{i}=(0.9 \pm 0.1) \cdot 10^{-7} R_{i}^{-1.0}$ and $R_{\max }^{v}-R_{i}=$ $(1.4 \pm 0.1) \cdot 10^{-7} R_{i}^{-1.0}$, and $V=(5.2 \pm 0.5) \cdot 10^{-6} R_{i}^{-1.0}$ and $V=(5.8 \pm 0.6) \cdot 10^{-6} R_{i}^{-1.0}$, for Solutions A1 and A3, respectively (lengths and times are given in meters and seconds). At longer times, the liquid reservoir empties and the whole system slowly drains back to its initial state. Again, no dependence on the solution viscosity could be detected during the shrinkage of the vertex (data not shown) in the range of parameters we investigated. We did not proceed further in the study of the deflation dynamics, which is partially ruled by gravitational drainage, and is certainly affected by finite size effects as the secondary jumps reach the frame within a few tens of milliseconds.

The characterization of the growth of the vertex gives some insights on its internal flow dynamics. While the viscosity ratio between the two solutions is equal to 9 , the ratio of the interpolations of the measured velocity is only 1.1 , and the ratio of the interpolations of the measured variation in size, $R_{\max }^{v}-R_{i}$, is about 1.6 . This shows that the viscosity does not play any significant role on the vertex dynamics and suggests that when the BP imbibition is inertial, the flow inside the vertex is inertial as well. However, the narrow range of parameters we have access to makes an extensive experimental study of the flow through a vertex very difficult.

The above results prompt two remarks. First, the exponents of the interpolations are difficult to retrieve within the framework of a dimensional analysis. Even though, this would be very difficult to test experimentally since, as already mentioned, we are not able to significantly vary the other parameters: density, surface tension, droplet size. The droplet size could not be varied over one decade without qualitatively changing the behavior observed, mainly because the droplet empties before the vertex has significantly grown. The second remark deals with the origin of dissipation in liquid foams. In the context of the drainage equation, it is now widely accepted that dissipation occurs mainly within the vertices when dealing with stress-free interfaces [7, 12], since one expects plug flows in the PBs and the shear zones to be located within the vertices. Our results question this conjecture as they show no evidence that the vertex prevails on the PB. This holds for the particular configuration we study, however this could also hold for liquid foams whose liquid fraction is not homogeneous, for instance in the zone of transition between the dry and the swollen regions observed in macroscopic drainage studies.

\section{B. Drainage through the bottom film}

In some cases, some of the liquid brought by the coalescing droplet flows through the vertical bottom film (see Fig. 1) instead of being evacuated within the PB. Fig. 9 illustrates this behavior for Solutions A1 and A6. A blob of liquid is observed to stretch downwards before eventually flowing, which is emphasized by the presence of a wake. A flow diagram is displayed in Fig. 10a, which reports the occurrence of the film drainage. Note that this occurrence is independent of the flow regime, inertial or viscous, inside the PB.

Within our parameters range, the drainage through the bottom film occurs for coalescing droplets having a radius larger than approximately $1 \mathrm{~mm}$, independently of the surfactant solution properties, as well as of the radius of curvature of the PB. This length is comparable to the capillary length. This suggests that gravity needs to be taken into account, which can be done by introducing the Bond number, $B o=\frac{\rho g r^{2}}{\gamma}$. Fig. $10 \mathrm{~b}$ shows a transition for a value of $B o$ about 0.36 .

\section{Influence of the surface mobility of the surfactant solutions}

Solution B was used to investigate effects of the surface mobility. This surfactant solution has the same low bulk viscosity and a surface viscosity significantly higher (by a factor 14) than Solution A1. Let's recall that we always observed the inertial regime with Solution A1. 


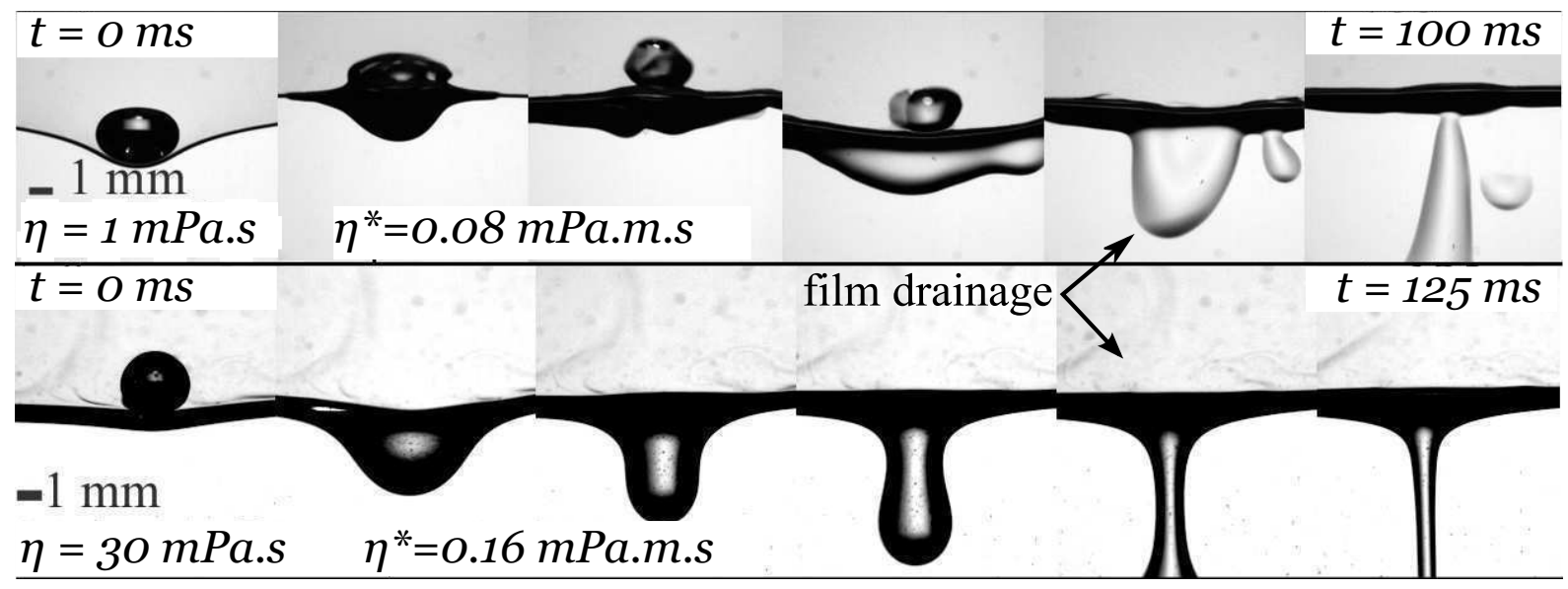

FIG. 9. Sequences of images that illustrate the film drainage for the low viscosity surfactant Solution A1 (top) and for Solution A6 thirty times more viscous than A1 (bottom).
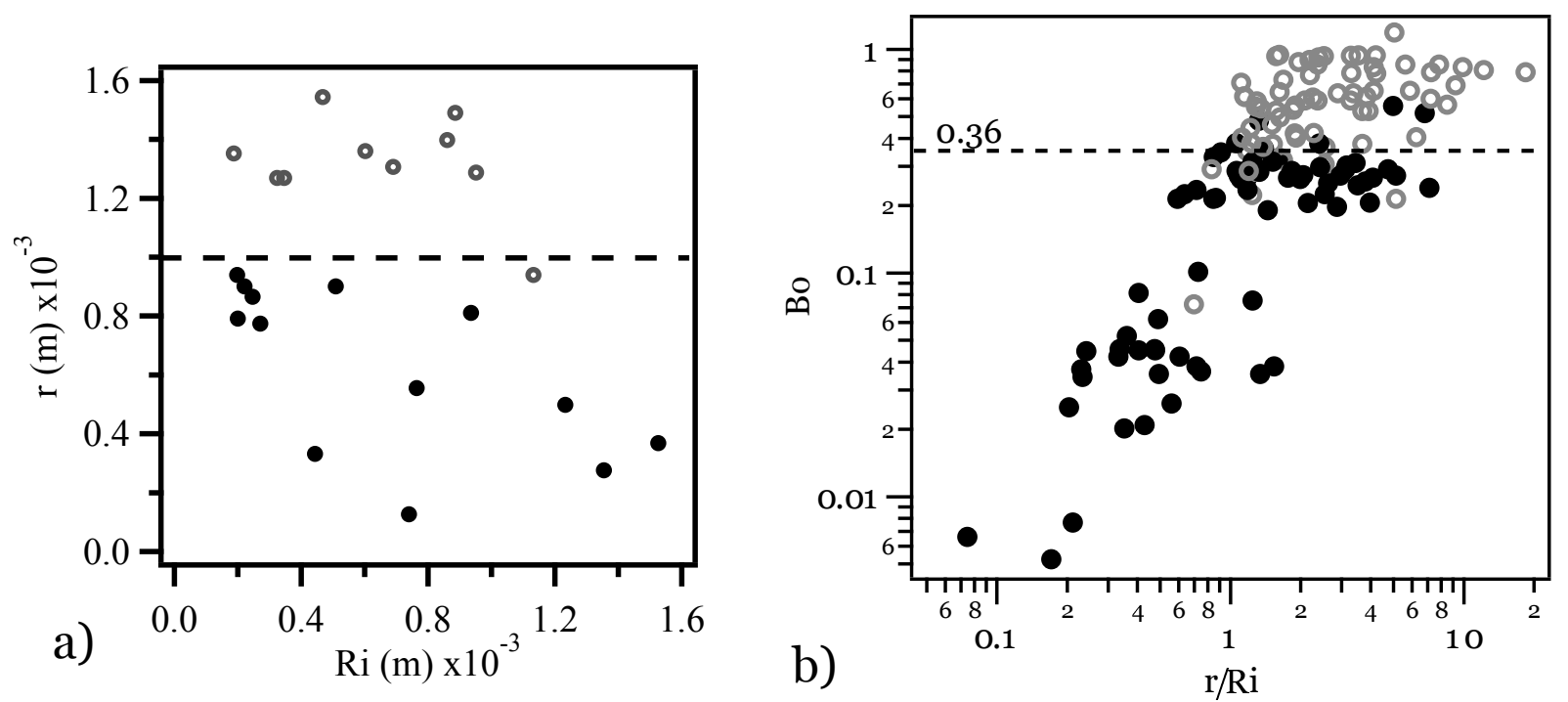

FIG. 10. Film drainage diagrams. The open symbols stand for the occurrence of film drainage while no film drainage was observed for filled symbols. a) $r-R_{i}$ diagram for Solution A3 $(\eta=9.2 \mathrm{mPa} . \mathrm{s})$. b) $B o-r / R_{i}$ diagram for all the measurements performed with Solutions A1 to A6. The dashed black line underlines a transition around $B o=0.36$.

The coalescence stage did not show any qualitative difference between Solution B and Solution A1. The data points in Figs. 2c and d follow the same trends as the ones obtained with the low surface viscosity solutions and the slight variation observed cannot be ascribed to the difference in surface mobility.

In contrast, the liquid redistribution stage differs strongly, as can be seen by comparing the time-evolutions reported in Figs. 3a and c. In the low surface mobility limit, the dynamics is characterized by the presence of a spatially extended front; the imbibition velocity, $c^{*}$, which was computed as before (see Sec. III C 3) as half of the time derivative of the spreading distance, is found to be constant, at least during the early stage of the liquid redistribution. We did not observe any correlation between the imbibition velocity and the droplet radius (insert of Fig. 11a), however Fig. 11a shows a clear dependency of $c^{*}$ on the initial radius of curvature of the $\mathrm{PB}, R_{i}$, as it is the case for the capillary inertial regime except that the opposite trend is obtained here, the larger the $\mathrm{PB}$ initial radius of curvature $R_{i}$, the higher the imbibition velocity $c^{*}$. Both quantities are roughly proportional, and a linear interpolation leads to $c^{*}$ $(\mathrm{m} / \mathrm{s})=(156 \pm 20) R_{i}(\mathrm{~m})$. This leads to Reynolds numbers larger than a few tens.

These results call for a comparison with the imbibition dynamics reported by Piroird and Lorenceau [21] for the suction of oil droplets by a single PB. Even though the setup geometries are analogous and the experiments performed with two similar surfactant solutions, both in the low surface mobility limit, distinct results and trends are obtained. In particular, the dynamics of the oil droplet imbibition is faster as $R_{i}$ decreases in contrast with our case (Figure 11). 




FIG. 11. Imbibition velocity $c^{*}$ (Solution B) as a function of the initial radius of curvature of the PB, $R_{i}$. The straight line on the graph is the best linear fit going through zero of the data points, and has a slope of $(156 \pm 20) \mathrm{s}^{-1}$. Insert : Imbibition velocity as a function of the droplet radius, $r$.

However, the mechanisms at play are expected to differ fundamentally between the two physical systems. Let's recall that Piroird and Lorenceau use silicon oils. First, these liquids are immiscible with the aqueous surfactant solution and the droplet creates an additional interface that alters the shape of the PB cross-section and thus the capillary pressure. Second, the silicon oils are much more viscous than the surfactant solution. Indeed Piroird and Lorenceau show that the elongation of an oil droplet inside a PB is a viscous process. In our case, the Reynolds numbers suggest that the flow might be inertia-dominated.

Another specific feature of the liquid redistribution for Solution B is noticeable on the last snapshot of Fig. 3c. For not too small droplets, namely droplets whose radius is larger than the radius of curvature of the PB, part of the liquid transfers during the coalescence process to the three films that hold the PB, and remains trapped there while only the remainder flows into the PB. Moreover, we hardly ever observed drainage through the bottom film (see Section IV B), even for the biggest droplets; consequently the criterion given above in the high surface mobility limit does not hold in the low surface mobility limit.

The surface viscosity of the surfactant solutions accounts for the interfacial stresses that oppose the interface dilatation and shear. This characteristic is expected to significantly influence the dynamics of the interface as the PB deforms and the holding films open, but also the dynamics of the bulk flow inside the PB, which strongly depends on the boundary conditions imposed at the interface [4]. Investigating the various effects of the surfactant mobility at the interfaces and rationalizing the experimental results would require to vary the surface characteristics of the surfactant solutions more systematically, which may be possible by playing on the physical chemistry of the surfactants; however, this is out of the scope of the present study.

\section{CONCLUSION}

We have designed a local scale experiment to study transient flows in liquid foams. This drop-injected experiment has yielded the following main results. First, we have shown that the coalescence of a droplet with a single Plateau border is inertia-dominated and its dynamics is similar to the dynamics of coalescence of a droplet with a liquid bath. Second, the imbibition process proves to be dominated either by inertia or viscosity; in the first case, its dynamics is controlled by the surface tension and the density of the surfactant solution, and the radius of curvature of the $\mathrm{PB}$, whereas the relevant parameters in the second case are the surface tension and the viscosity of the surfactant solution, and the radius of the coalescing droplet. The liquid redistribution may actually switch from the capillary inertial regime to the viscous one in the course of an experiment. We propose to rationalize this transition assuming a velocity-limiting mechanism.

We have shown that the interfacial rheology plays a major role on the regime that is inertia-dominated in the high 
surface mobility limit. This issue would need to be further investigated.

In the future, occurrence of the inertia-dominated regime triggered by capillary suction needs to be investigated at the foam scale. Microgravity experiments might be an option [14, 15]; a close look at the front zone in drainage experiments $[22,23]$ could also disclose evidences of the capillary inertial regime.

\section{ACKNOWLEDGMENTS}

We gratefully acknowledge fruitful discussions with Jean Rajchenbach, Médéric Argentina and Yann Bouret. Elise Lorenceau is thanked for insightful comments. This work was supported by a research funding granted by the University of Nice Sophia Antipolis.

[1] R. K. Prud'home and S. A. Khan. In foams: Theory, measurements, and applications. Marcel Dekker Inc., New York, 1996.

[2] D. Weaire and S. Hutzler. The Physics of Foams. 1999.

[3] I. Cantat, S. Cohen-Addad, F. Elias, F. Graner, R. Höhler, O. Pitois, F. Rouyer, and A. Saint-Jalmes. Foams: structure and dynamics. Oxford University Press, Oxford, 2013.

[4] A. Saint-Jalmes. Physical chemistry in foam drainage and coarsening. Soft Matter 2, pages 836-849, 2006.

[5] D. Weaire, N. Pittet, S. Hutzler, and D. Pardal. Steady-state drainage of an aqueous foam. Phys. Rev. Lett., 71:2670, 1993.

[6] G. Verbist and D. Weaire. A soluble model for foam drainage. Europhys. Lett., 26:631-634, 1994.

[7] S A. Koehler, S. Hilgenfeldt, and H. A. Stone. A generalized view of foam drainage: Experiment and theory. Langmuir, 16:6327-6341, 2000.

[8] S. Cohen-Addad, R. Höhler, and O. Pitois. Flow in foams and flowing foams. Annu. Rev. Fluid Mech., 45:241-267, 2013.

[9] M. In Het Panhuis, S. Hutzler, D. Weaire, and R. Phelan. New variations on the soap film experiments of plateau i. experiments under forced drainage. Philosophical Magazine Part B, 78(1):1-12, 1998.

[10] S.A. Koehler, S. Hilgenfeldt, E.R. Weeks, and H.A. Stone. Foam drainage on the microscale ii. imaging flow through single plateau borders. Journal of colloid and Interface Science, 276:439-449, 2004.

[11] O. Pitois, C. Fritz, and M. Vignes-Adler. Hydrodynamic resistance of a single foam channel. Colloids and Surfaces A: Physicochemical and Engineering Aspects, 261:109-114, 2005.

[12] S. A. Koehler, S. Hilgenfeldt, and H. A. Stone. Liquid flow through aqueous foams: The node-dominated foam drainage equation. Phys. Rev. Lett., 82:4232, 1999.

[13] A. Saint-Jalmes, Y. Zhang, and D. Langevin. Quantitative description of foam drainage: Transitions with surface mobility,. Eur. Phys. J. E, 15:53-60, 2004.

[14] H. Caps, S.J. Cox, H. Decauwer, D. Weaire, and N. Vandewalle. Capillary rise in foams under microgravity. Colloids Surf. A, 261:131-134, 2005.

[15] A. Saint-Jalmes, S. Marze, H. Ritacco, D. Langevin, S. Bail, J. Dubail, L. Guingot, G. Roux, P. Sung, and L. Tosini. Diffusive liquid propagation in porous and elastic materials: The case of foams under microgravity conditions. Phys. Rev. Lett, 98:058303, 2007.

[16] A. Cohen, N. Fraysse, J. Rajchenbach, M. Argentina, Y. Bouret, and C. Raufaste. Inertial mass transport and capillary hydraulic jump in a liquid foam microchannel. Phys. Rev. Lett., 112:218303, 2014.

[17] M. Argentina, A. Cohen, Y. Bouret, N. Fraysse, and C. Raufaste. One dimensional capillary jumps. JFM, 2014.

[18] K. Golemanov, N. D. Denkov, S. Tcholakova, M. Vethamuthu, and A. Lips. Surfactant mixtures for control of bubble surface mobility in foam studies. Langmui, 24:9956-9961, 2008.

[19] A. L. Biance, S. Cohen-Addad, and R. Höhler. Topological transition dynamics in a strained bubble cluster. Soft Matter, $5: 4672-4679,2009$

[20] S.T. Thoroddsen and K. Takehara. The coalescence cascade of a drop. Phys. Fluids, 12:1265, 2000.

[21] K. Piroird and E. Lorenceau. Capillary flow of oil in a single foam microchannel. Phys. Rev. Lett., 111:234503, Dec 2013.

[22] P. Grassia, J.J. Cilliers, S.J. Neethling, and E. Ventura-Medin. Quasione-dimensional foam drainage. Eur. Phys. J. E, 6:325-348, 2001.

[23] P. Grassia, S.J. Neethling, and J.J. Cilliers. Foam drainage on a sloping weir. Eur. Phys. J. E, 8:517-529, 2002. 